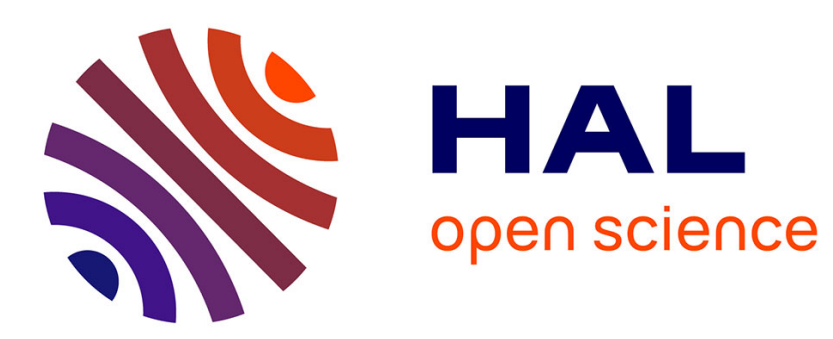

\title{
Spectroscopie des composés Ga1-xAlxAs par modulation de longueur d'onde
}

\author{
R. Lande, R. Madelon, A. Hairie, A. Fortini
}

\section{To cite this version:}

R. Lande, R. Madelon, A. Hairie, A. Fortini. Spectroscopie des composés Ga1-xAlxAs par modulation de longueur d'onde. Revue de Physique Appliquée, 1977, 12 (3), pp.483-485. 10.1051/rphysap:01977001203048300 . jpa-00244200

\section{HAL Id: jpa-00244200 https://hal.science/jpa-00244200}

Submitted on 1 Jan 1977

HAL is a multi-disciplinary open access archive for the deposit and dissemination of scientific research documents, whether they are published or not. The documents may come from teaching and research institutions in France or abroad, or from public or private research centers.
L'archive ouverte pluridisciplinaire HAL, est destinée au dépôt et à la diffusion de documents scientifiques de niveau recherche, publiés ou non, émanant des établissements d'enseignement et de recherche français ou étrangers, des laboratoires publics ou privés. 
Classification

Physics Abstracts

$8.824-0.644$

\title{
SPECTROSCOPIE DES COMPOSÉS Ga 1-x $\mathrm{Al}_{x} \mathrm{AS}$ PAR MODULATION DE LONGUEUR D'ONDE (*)
}

\author{
R. LANDE, R. MADELON, A. HAIRIE et A. FORTINI \\ Laboratoire de Physique du Solide de l'Université de Caen, 14032 Caen Cedex, France
}

(Reçu le 25 octobre 1976, accepté le 3 décembre 1976)

\begin{abstract}
Résumé. - On a effectué des mesures de réflectivité par modulation de longueur d'onde entre 1,3 et $5,5 \mathrm{eV}$ sur des couches épitaxiales de $\mathrm{Ga}_{1-x} \mathrm{Al}_{x} \mathrm{As}$ de composition variable $0<x<0,62$. Les résultats, qui sont en bon accord avec les mesures antérieures d'électroréflectance sur ces composés, prouvent que cette méthode de modulation peut être d'un grand intérêt malgré ses difficultés techniques.
\end{abstract}

\begin{abstract}
Reflectivity measurements by wavelength modulation have been performed from 1.3 to $5.5 \mathrm{eV}$ on epitaxial layers of $\mathrm{Ga}_{1-x} \mathrm{Al}_{x} \mathrm{As}$ with different compositions $(0<x<0.62)$. The results, in good agreement with earlier electroreflectance measurements on these compounds, prove the great interest of this method in spite of its technical difficulties.
\end{abstract}

1. Introduction. - L'analyse de la structure de bande des composés semi-conducteurs par spectroscopie différentielle s'est considérablement développée au cours des dernières années. Il existe différentes méthodes pour obtenir la dérivée d'un spectre de transmission ou de réflexion. Leurs avantages et leurs inconvénients sont discutés dans l'ouvrage de M. Cardona [1]. La modulation externe de longueur d'onde qui est, du moins en principe, la plus simple et la plus naturelle, a été beaucoup moins utilisée que les méthodes de modulation interne, techniquement plus faciles à mettre en œuvre. Elle présente néanmoins l'avantage primordial de fournir la dérivée du spectre directement plutôt que par l'intermédiaire de la dérivée d'une propriété du matériau.

Les composés binaires III-V et pseudobinaires III-III'-V et III-V-V' ont été surtout étudiés en électroréflectance $[2,3,4,5]$ piézoréflectance [6], thermoréflectance [7]. Dans les pseudobinaires, les résultats de spectroscopie présentent habituellement une évolution régulière des singularités de Van Hove avec la composition.

C'est ce dernier type d'étude que nous avons entrepris sur les composés $\mathrm{Ga}_{1-x} \mathrm{Al}_{x} \mathrm{As}$, en modulation de longueur d'onde. Ces composés, qui sont d'un grand intérêt technique, ont été intensivement étudiés en électroluminescence, cathodoluminescence et photo-

(*) Cette étude a été effectuée avec le soutien de la D. G. R. S. T. (contrat no 73-7-1168). luminescence $[8,9,10,11,12]$, mais n'ont fait l'objet que d'une seule étude en spectroscopie différentielle, par électroréflectance [2].

Le spectromètre à modulation de longueur d'onde réalisé à Caen se caractérise par un dédoublement temporel du faisceau sur une faible partie de sa longueur, ce qui assure l'élimination presque parfaite des structures dérivées parasites. L'appareil fournit la dérivée du spectre par voie analogique. Il doit faire l'objet d'une publication ultérieure.

2. Préparation des échantillons et résultats. - Les échantillons de $\mathrm{Ga}_{1-x} \mathrm{Al}_{x} \mathrm{As}$ ont été fabriqués à la Radiotechnique-Compelec, Usine de Caen, par croissance épitaxiale en phase liquide, sur substrat de GaAs. L'épaisseur des couches était de l'ordre de 40 à $50 \mu$, les dimensions transversales utiles d'environ $5 \mathrm{~mm}$. La composition superficielle en aluminium a été mesurée à la sonde de Castaing au Centre National d'Etudes et Télécommunications, Laboratoire de Bagneux.

L'observation de structures en réflexion était largement tributaire du traitement de surface des échantillons. Les meilleurs résultats ont été obtenus après un polissage chimique de 15 secondes dans la solution $3 \mathrm{SO}_{4} \mathrm{H}_{2}+1 \mathrm{H}_{2} \mathrm{O}_{2}+1 \mathrm{H}_{2} \mathrm{O}$, à $50^{\circ} \mathrm{C}$, environ, suivi d'un rinçage à l'eau distillée, puis à l'alcool absolu.

Les spectres de réflexion dérivée obtenus à $80 \mathrm{~K}$ sont représentés sur les figures 1 et 2 . Les singularités de Van Hove sont indiquées à l'aide des notations habituelles, rappelées figure 3 , sur la structure de 


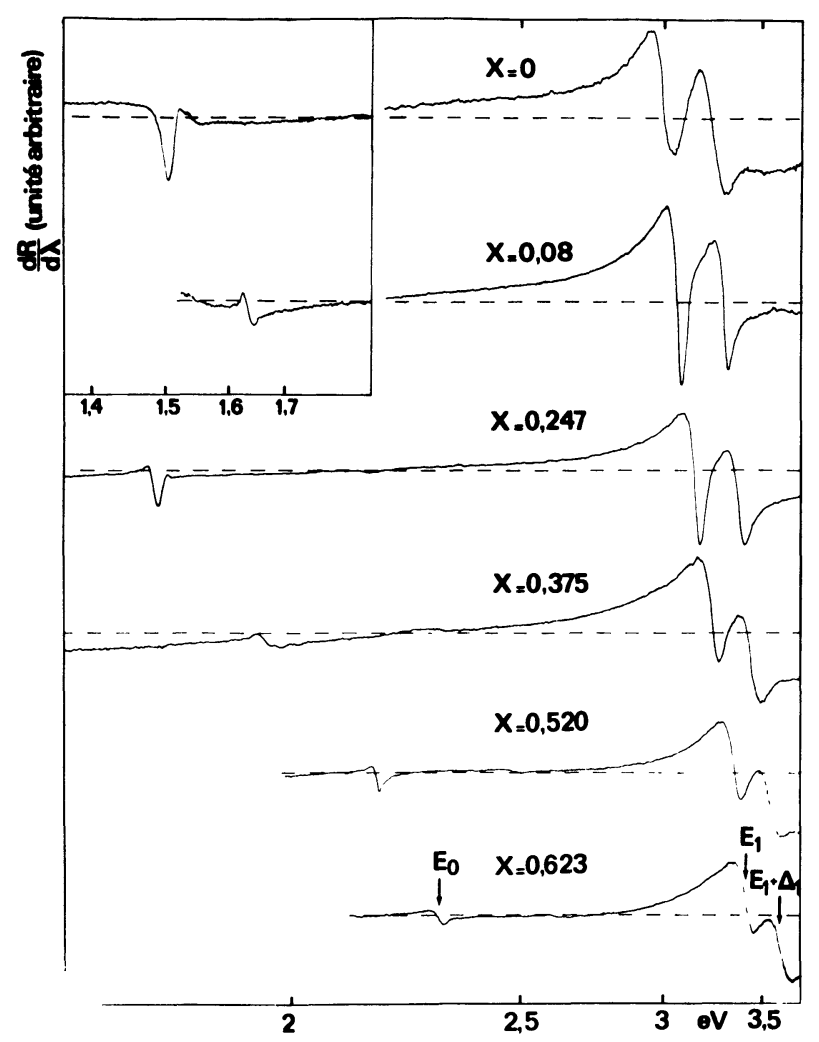

Fig. 1. - Dérivée du spectre de réflectivité de composés $\mathrm{Ga}_{1-x} \mathrm{Al}_{x} \mathrm{As}$ entre $1,4 \mathrm{et} 3,6 \mathrm{eV}$, à $80 \mathrm{~K}$, pour différentes valeurs de la composition en aluminium $(x)$.

[Derivative of the reflectivity spectrum of $\mathrm{Ga}_{1-x} \mathrm{Al}_{x} \mathrm{As}$ from 1.4 to $3.6 \mathrm{eV}$ at $80 \mathrm{~K}$, for different values of the aluminium concentration $x$.]

bande de GaAs. La concentration d'aluminium est indiquée directement sur les courbes.

Ces résultats sont en bon accord avec ceux obtenus en modulation de longueur d'onde par R. Zucca, J. Walter, Y. Shen et M. Cohen [13] sur GaAs, à $5 \mathrm{~K}$, et ceux obtenus en électroréflectance sur GaAlAs à $300 \mathrm{~K}$ par $\mathrm{O}$. Berolo et J. C. Wooley [2].

On observe, sur la figure 1, l'évolution de la transition fondamentale $E_{0}$ depuis la valeur $1,51 \mathrm{eV}$ dans GaAs $(x=0)$. Le dédoublement spin-orbite de cette transition $\left(E_{0}+\Delta_{0}\right)$ n'est pas observé, sans doute à cause de la moindre densité d'états dans la bande $\Gamma_{7}$. Les structures $E_{1}$ et $E_{1}+\Delta_{1}$ qui apparaissent plus nettement se déplacent beaucoup moins avec la composition.

Le pic observé vers 4,5 eV (Fig. 2) peut être dû aux transitions $\Gamma_{15}-\Gamma_{15}, X_{5}-X_{3}$ où à des transitions dans la direction $\Delta$. Suivant Zucca [13], nous l'avons attribué à ces dernières.

Enfin, le pic intense vers $5 \mathrm{eV}$ (Figure 2) provient [14] d'une transition $\Sigma_{2}-\Sigma_{1}$ dans la direction 110 , dont la force d'oscillateur est très grande.

Les résultats obtenus dans la gamme 4,3-5,7 eV sont dans l'ensemble plus confus qu'au voisinage des transitions $E_{0}$ et $E_{1}$. Ceci peut s'expliquer par les plus grandes difficultés expérimentales rencontrées en ultra-violet où le rôle de la surface devient parti-

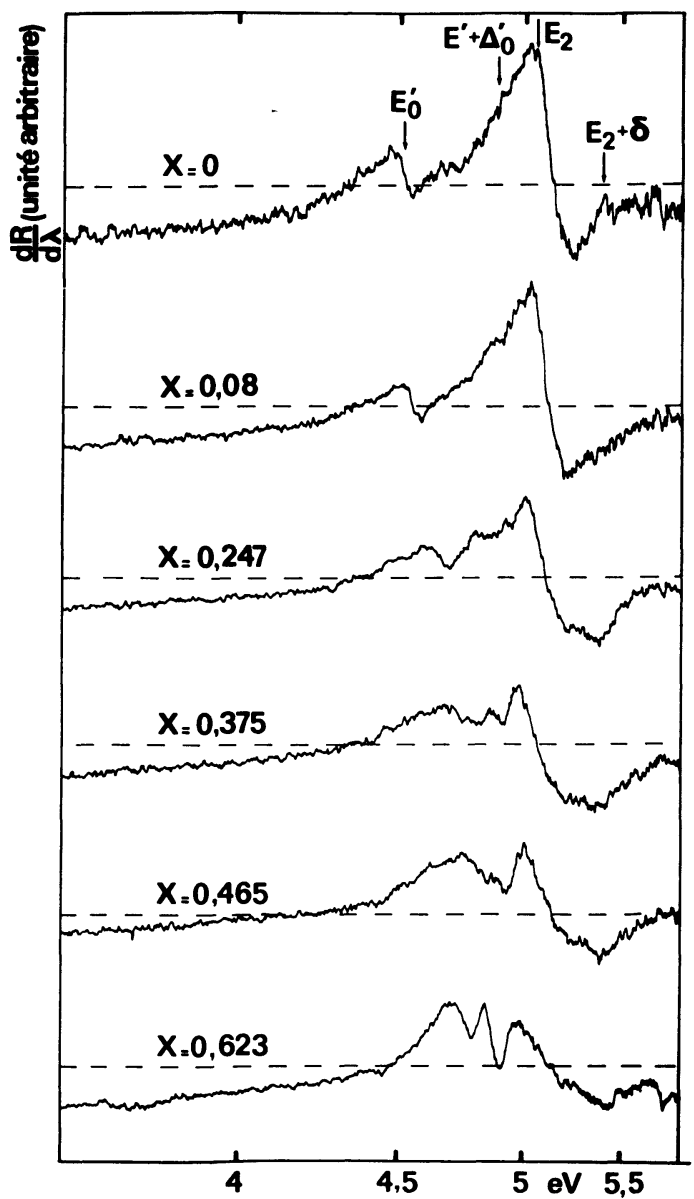

FIG. 2. - Dérivée du spectre de réflectivité des composés $\mathrm{Ga}_{1-x} \mathrm{Al}_{x} \mathrm{As}$ entre 3,5 et $5,5 \mathrm{eV}$, à $80 \mathrm{~K}$, pour différentes valeurs de la composition $x$.

[Derivative of the reflectivity spectrum of $\mathrm{Ga}_{1-x} \mathrm{Al}_{x} \mathrm{As}$ from 3.5 to $5.5 \mathrm{eV}$ at $80 \mathrm{~K}$, for different values of the aluminium concentration $x$.]

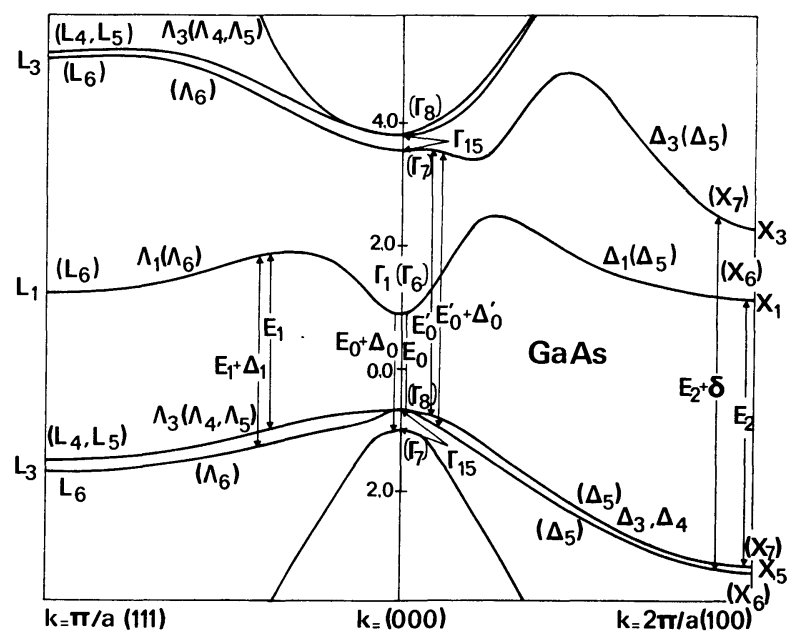

FIG. 3. - Structure de bande de GaAs dans les directions 100 et 111 de la zone de Brillouin, d'après F. H. Pollak, C. W. Higginbotham et M. Cardona [16].

[Band structure of GaAs in the directions [100] and [111] of the Brillouin Zone, from F. H. Pollak, C. W. Higginbotham and M. Cardona [16].]

culièrement critique ; en outre, un grand nombre de transitions sont susceptibles de contribuer à la structure du spectre dans cette zone. 


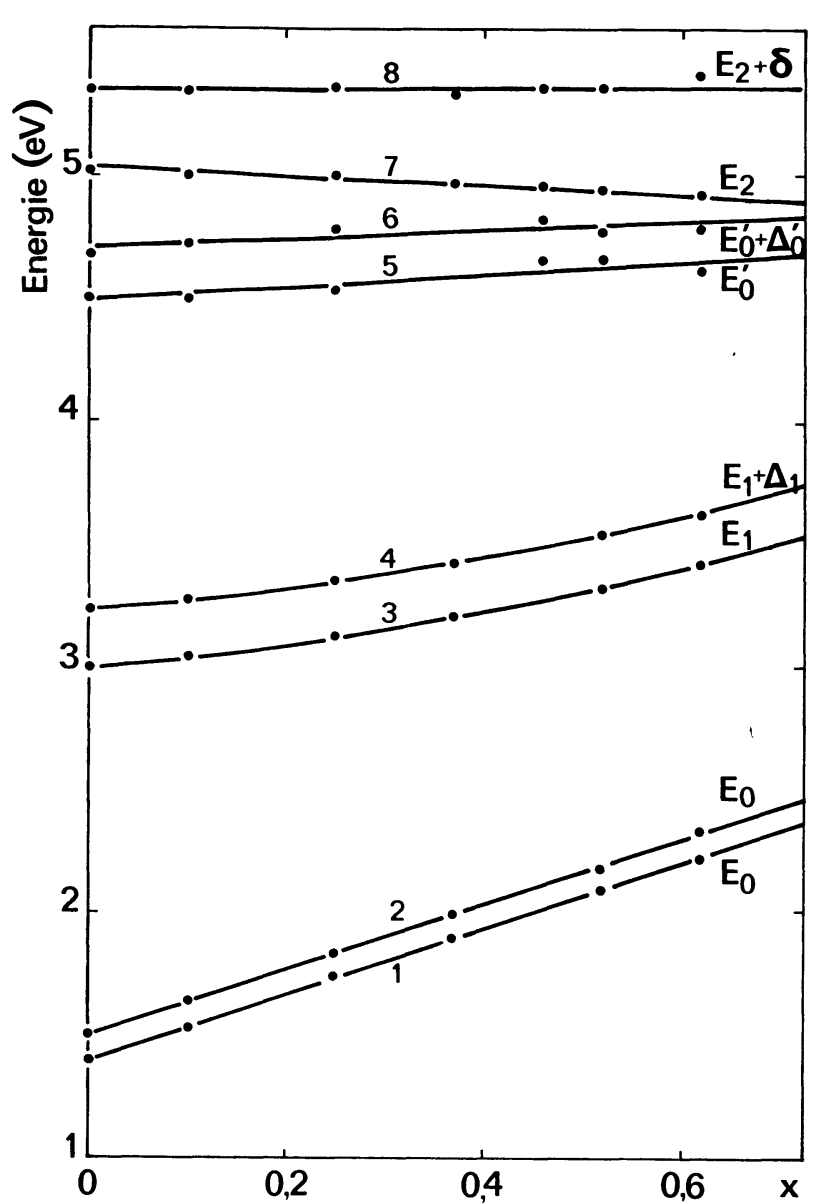

Fig. 4. - Variations de la position en énergie des singularités de Van Hove, dans les composés $\mathrm{Ga}_{1-x} \mathrm{Al}_{x} \mathrm{As}$, en fonction de la composition $x$.

[Variation of the energy of Van Hove singularities in $\mathrm{Ga}_{1-x} \mathrm{Al}{ }_{x} \mathrm{As}$ compounds, as a fonction of composition.]

Les déplacements des différentes singularités observées, en fonction de la composition $x$, sont représentés figure 4. L'énergie $E_{0}$ (en $\mathrm{eV}$ ) de la transition fondamentale $\Gamma_{8}-\Gamma_{6}$, croît linéairement avec $x$ selon :

$$
\begin{array}{ll}
E_{0}=1,50+1,33 x & \text { à } 80 \mathrm{~K} \\
E_{0}=1,40+1,34 x & \text { à } 300 \mathrm{~K}
\end{array}
$$

en accord satisfaisant avec Casey et Panish [15] et Berolo et Wooley [2] bien qu'il n'apparaisse pas ici de courbure, dans les limites d'erreur expérimentale, comme l'ont signalé ces auteurs.
Pour les transitions $\Lambda_{3}-\Lambda_{1}$ l'évolution en fonction de $x$ est par contre, légèrement parabolique. On a obtenu (en eV) :

$$
\begin{aligned}
E_{1}+\Delta E_{1} & =3,23+0,375 x+0,43 x^{2} \text { à } 80 \mathrm{~K} . \\
E_{1} & =3,00+0,37 x+0,49 x^{2} \text { à } 80 \mathrm{~K} .
\end{aligned}
$$

Cette fois, le paramètre de courbure est en accord avec la valeur moyenne de 0,45 donnée par Berolo et Wooley [2].

Dans la région 4,5-6 eV, les résultats sont moins nets pour les raisons données ci-dessus. Seule la transition $\Sigma_{2}-\Sigma_{1}$ (énergie $E_{2}$ ) est toujours bien visible. On constate sur la figure 4 que l'énergie correspondante $E_{2}$ est une fonction linéaire, légèrement décroissante de $x$.

On a également représenté, figure 3, l'énergie $E_{2}+\delta$ de la transition $X_{5}-X_{3}$, pratiquement indépendante de $x$, ainsi que les énergies $E_{0}^{\prime}$ et $E_{0}^{\prime}+\Delta_{0}^{\prime}$ des transitions $\Gamma_{15}-\Gamma_{15}$, grossièrement linéaires avec $x$.

Ces différentes structures ont aussi été observées par Berolo et Wooley [2] en électroréflectance.

3. Conclusion. - Les résultats que nous avons obtenus dans l'étude des singularités de Van Hove des composés $\mathrm{Ga}_{1-x} \mathrm{Al}_{x} \mathrm{As}$, en fonction de $x$, sont en bon accord avec ceux de Berolo et Wooley obtenus en électroréflectance et de Matatagui et Thompson [7] en thermoréflectance.

L'absence de courbure dans la dépendance en $x$ de la transition fondamentale $E_{0}$ serait en accord qualitatif avec la théorie de Van Vechten [15], mais dans l'ensemble les résultats obtenus appellent de nouveaux développements théoriques.

Au stade présent, l'intérêt de cette étude se situe essentiellement sur le plan technique puisqu'elle a prouvé que, moyennant quelques raffinements dans la mise au point du spectromètre, la modulation externe de longueur d'onde, qui offre des commodités opératoires indéniables, est tout à fait compétitive avec les autres techniques de modulation.

Remerciements. - Les auteurs remercient la Radiotechnique Compelec, à Caen, qui a fourni gracieusement les couches épitaciales étudiées, et le Centre National d'Etudes des Télécommunications, Laboratoire de Bagneux, qui a bien voulu accepter de mesurer la concentration superficielle d'aluminium.

\section{Bibliographie}

[1] Cardona, M., "Modulation Spectroscopy " (Acad. Press. N. Y. and London).

[2] Berolo, O. and Woolley, J. C., Can. J. Phys. 49 (1971) 1335.

[3] Thompson, A. G., Cardona, M., Shaklee, K. L. and Woolley, J. C., Phys. Rev. 146 (1966) 601.

[4] Cardona, M., Shaklee, K. L. and Pollak, F. H., Phys. Rev. 154 (1966) 696.

[5] Vishnubhatla, S. S., Eyglunent, B. and Wooley, J. C., Can. J. Phys. 47 (1969) 1661.

[6] Auvergne, D., Merle, P., Zein-Eldin, A., Mathieu, H. and Nguyen Van Mau, A., Sol. Stat. Commun. 17 (1975) 511

[7] Matatagui, E., Thompson, A. G. and Cardona, M., Phys. Rev. 176 (1968) 950

[8] Kressel, H., Nicoll, F. H., Hawrylo, F. Z. and Lockwood, H. F., J. Appl. Phys. 41 (1970) 4692
[9] Alferov, Zh. I., Garbuzov, Dz., Kolyshin, V. I. Ninua, O. A. and Trofim, V. G., Sov. Phys. Semicond. 5 (1972) 1228 et 1232.

[10] Jagdeep Shah, Miller, B. I. and Digiovanni, A. E. J. Appl. Phys. 43 (1972) 3436.

[11] Shun-IChI-Gowda and Yvnosuke Makita, Appl. Phys. Lett. 27 (1975) 392.

[12] Bumelis, A. J., Maldutis, E. K., Matulenis, A. Yu., Shimkyavichus, Ch. I., Shimulite, E. A., Sov. Phys. Semi-cond. 9 (1976) 1081.

[13] Zucca, R., Walter, J., Shen, Y. and Cohen, M., Solid State Commun. 8 (1970) 627.

[14] Marcus gray, A., Phys. Stat. Sol. 37 (1970) 11.

[15] Van Vechten, J. A. and Bergstresser, T. K., Phys. Rev. 1 (1970) 3351.

[16] Pollak, F. H., Higginbotham, C. W. and Cardona, M., J. Phys. Soc. Japan Suppl. 21 (1966) 20. 